\title{
Generalized Extremal Optimization Algorithm to design a LED-based spectrally tunable light source for Star Simulation
}

\author{
Marcos E.G. Borges ${ }^{1}$ and Lamartine N.F. Guimarães ${ }^{2}$ \\ Manuscript received on November 2, 2013 / accepted on December 16, 2014
}

\begin{abstract}
An Autonomous Star Tracker (AST) has been designed and is being constructed at National Institute for Space Research (INPE). In order to calibrate and characterize the AST a sophisticated infrastructure of tests is required. This infrastructure, composed of several instruments, has included one Star Simulator (SS). The simulator has also been designed and constructed at INPE. The SS is basically composed of a LED-based spectrally tunable light source, a pinhole and a collimator. The SS is designed to have the capability of producing different continuum spectral distributions, mimicking star magnitudes in the visible and near-infrared range, what is achieved through the feedback control of individual LEDs. The objective of this work is to present a methodology for the optimal selection of the LEDs that compose the light source. For that, an evolutionary multi-objective approach is used. This approach tries to get the best spectral simulation and at the same time to reduce the number of LEDs used. The methodology applied to model the stars is also presented in this paper, together with a theoretical basis applied to model the LEDs. A series of simulations have been conducted to predict the performance of the designed tunable source. Many source distributions have been constructed for a number of target distributions, and the results are promising.
\end{abstract}

Keywords: Autonomous Star Tracker, light source, LED, optimization, generalized extremal optimization.

\section{INTRODUCTION}

The Autonomous Star Tracker (AST) is an electro-optical equipment applied to three-axis attitude determination of space or suborbital vehicles. It makes use of star positions as a setting reference, whose relative directions about the Earth are considered to be constant. The attitude determination corresponds to identify the space zone towards which the sensor is aimed at [1, 2]. For that, the sensor acquires and processes images to determine the stars present in the field of view, and thus to calculate the attitude.

In order to calibrate, test and characterize the AST, a sophisticated infrastructure known as Ground Support Equipment (GSE) is required. This infrastructure, composed of several equipments, simulates stars for the optical calibration of the AST with the Star Simulator (SS). The simulator in question is composed of one LED-based Spectrally Tunable Light Source (STLS), a pinhole onto its focal plane and a parabolic collimator out of axis.

The spectral simulation is frequently necessary for several radiometric and photometric tasks. In the present work it has been applied in the generation of star spectra required for the calibration and tests of the AST. By using light emitting diodes (LEDS) with different spectral power distributions (SPDs) and controlling the radiometric output of each type of LED, it is possible to make a spectrally tunable light source capable of producing a high fidelity spectral match to a specific "target" SPD [3].

Correspondence to: Marcos E.G. Borges - E-mail: marcosegborges@gmail.com

${ }^{1}$ Postgraduate Program in Applied Computing, National Institute for Space Research (INPE), São José dos Campos, SP, Brazil.

${ }^{2}$ Nuclear Energy Division, Institute for Advanced Studies (IEAv), São José dos Campos, SP, Brazil. 
The STLS which uses an integrating sphere and a large number of LEDs has been designed and constructed in the Aerospace Electronics Division of INPE. The design and construction of this source of light displayed several challenges. Among them the definition of the necessary quantity and types of LEDs to make possible the simulation of stars with magnitudes from zero to five, besides the characterization and calibration of the AST.

The choice of quantity and types of LEDs for the STLS to be able to simulate the detectable stars through AST is a multiobjective optimization problem. The objective is to minimize the quantity of LEDs and at same time maximize the spectral matching capability of the star spectra. The main aim of this work is to present the methodology that has been applied using the Generalized Extremal Optimization algorithm (GEO) for optimally choosing the quantity and types of LEDs to perform the proposed task.

This paper is organized as follows: Section 2 presents the architecture of the SS and all equations necessary to model the star spectra. Section 3 presents information on the construction and architecture of the STLS. It also presents the equations used to model the LEDs. Section 4 deals with the multi-objective approach, presenting the GEO algorithm and its multi-objective version, the M-GEO. Section 5 presents the methodology applied for the optimal choice of LEDs. Section 6 presents the results and Section 7 the conclusions.

\section{DESIGN OF THE STAR SIMULATOR}

For the characterization, tests and calibration of the AST, a sophisticated infrastructure, the GSE, is necessary. Among several items that compose the GSE, there is the SS. The SS generates similar electromagnetic continuum spectra as those of the stars, for the AST optical calibration.

The SS is composed of a STLS, one out of axis parabolical collimator and one pinhole onto its focal position. The STLS generates spectra similar to those emitted by stars. The light beam generated by the source will go through the pinhole orifice and will be projected on the collimator. The collimator has the function to turn into parallel the divergent luminous beam received through the pinhole, simulating a source of light in the infinity, that is, simulating the irradiation emitted by a star. Figure 1(a) displays the integrating sphere with the LED heads. Figure 1(b) displays the SS with its main components.

\subsection{Star Magnitudes}

The AST will operate to identify stars into the range of visual magnitudes from zero to five $[4,5]$. The magnitudes of stars are com- parative quantities represented by a logarithmic scale. The visual magnitude is defined by the following equation [6]:

$$
m_{v 1}-m_{v 2}=2.5 \log \left(\frac{I_{2}}{I_{1}}\right),
$$

where $m_{v 1}$ and $m_{v 2}$ are the visual magnitudes of two different stars, and $I_{1}$ and $I_{2}$ their respective spectral irradiances. The visual magnitude of a star is equal to zero when its spectral irradiance outside the Earth's atmosphere is $4 \times 10^{-9} \mathrm{erg} \mathrm{cm}^{-2} \mathrm{~s}^{-1} \AA^{-1}$ at $550 \mathrm{~nm}$ [6], or equivalently $4 \times 10^{-15} \mathrm{~W} \mathrm{~cm}^{-2} \mathrm{~nm}^{-1}$.

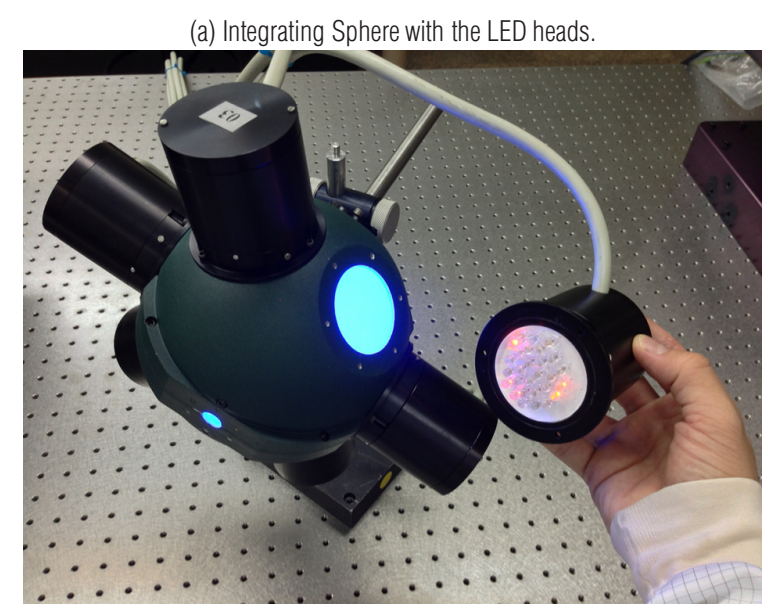

(b) Star Simulator.

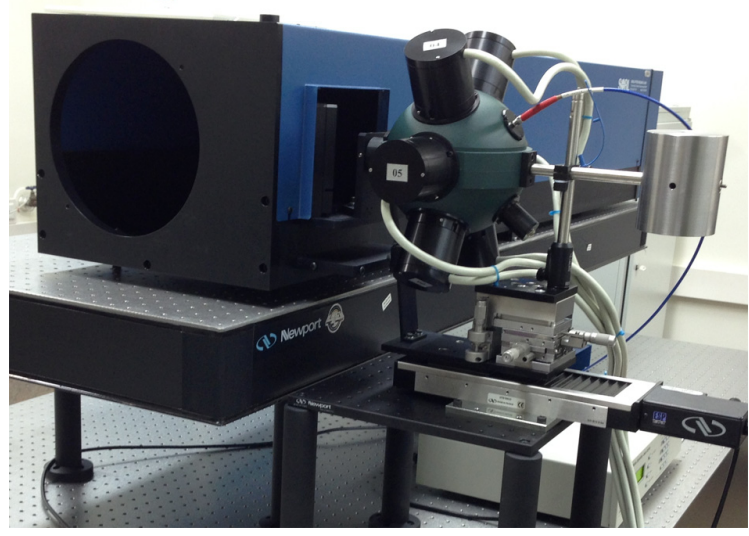

Figure 1 - Configuration of the INPE Star Simulator.

\subsection{Star Spectral Irradiance}

The spectral irradiance of a star can be modeled by a black body spectral power distribution $[6,7]$. A black body is an object that absorbs the entire electromagnetic radiation that falls into it. No radiation passes through it and none is reflected $[6,7,8,9,10]$. The radiation spectrum of a black body is characterized by an unique parameter, $T$, the temperature of the black body. The black body spectral irradiance was derived for the first time by Max 
Plank (1900) $[6,7,10]$, and is given by:

$$
I(\lambda)=\frac{2 h c^{2}}{\lambda^{5}\left[\exp \left(\frac{h c}{\lambda k T}\right)-1\right]} .
$$

The unit of $I(\lambda)$ is $\mathrm{W} \mathrm{m}^{-2} \mathrm{~nm}^{-1}$ and thus it represents the emitted energy by an unit of surface area $\left(\mathrm{m}^{2}\right)$ of the black body per unit of wavelength $\lambda$ (nanometer). $T$ is the temperature of the black body in Kelvin (K), $k$ is Boltzmann's constant $(\mathrm{J} / \mathrm{K}), h$ is Planck's constant $(\mathrm{J} \mathrm{s})$ and $c$ is the speed of light $(\mathrm{m} / \mathrm{s})$.

The black body continuum spectrum depends on its temperature $[6,7,8,9,10]$. The star temperature levels that the AST will identify has been obtained by a statistic analysis of star temperatures. The Hipparcos Stars Catalogue $[11,12]$ has been utilized for this task. Only stars of apparent magnitude from zero to five have been selected, resulting in a total of 1625 stars. The catalogue does not displays information about the star temperatures. The approximate effective temperature of each star was calculated using the empirical equations proposed by [13], that have their basis on the Johnson B-V color index.

$$
\begin{aligned}
T= & 10^{(3.402-\sqrt{0.515+1.376(B-V)}) / 0.688} \\
& \text { for }-0.375<(B-V)<-0.041, \\
T= & 10^{((B-V)-14.551) /-3.684} \\
& \text { for }(B-V)>=-0.041,
\end{aligned}
$$

where $T$ is the temperature in Kelvin, (B) and (V) are special photometric filters utilized in the composition of the Johnson color index. Each filter is positioned at a specific band of the electromagnetic spectrum: (B) centered at $445 \mathrm{~nm}$ (blue region) and (V) centered at $557 \mathrm{~nm}$ (visible region) [11].

The spectral characteristic of the light source is very important to simulate stars in an appropriate manner. Studies about stars spectral characteristics at the interval of magnitude that the AST will operate were conducted by [4]. Figure 2 shows the histogram with the distribution of star temperatures in the used sample in the magnitude range from zero to five. Most stars present temperatures between $3000 \mathrm{~K}$ to $20000 \mathrm{~K}$.

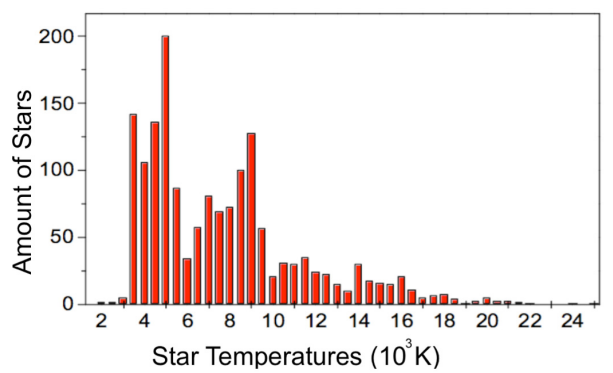

Figure 2 - Histogram for the temperatures of the sampled stars from Hipparcos Stars Catalogue.

\subsection{Spectral Irradiance in the Collimator Output}

By using Equations 1, 2 and 3a it is possible to model any star knowing its temperature and magnitudes. To simulate stars knowing their spectral irradiance calculated by the previous equations, it is necessary to know the spectral irradiance in the collimator output. Figure 3 shows the details of how the integrating sphere is used together with the pinhole and collimator to simulate the continuous spectrum of stars.

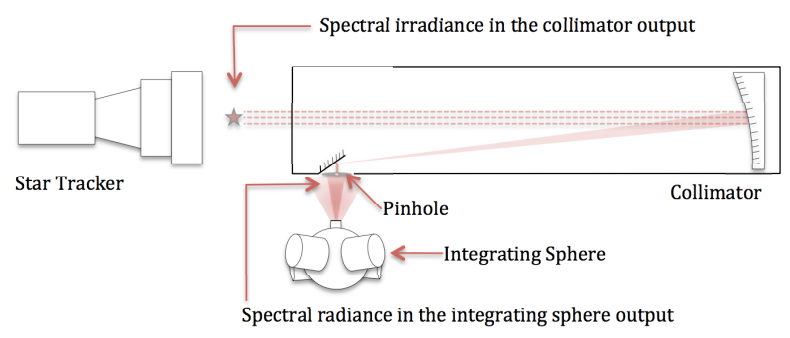

Figure 3 - Simulation of a star spectral irradiance in the Star Simulator.

Over the simulation process, the integrating sphere generates on its output, a spectral radiance from the combination of the spectra produced by multiple LEDs at different powers. The spectral radiance generated by the source will go through the pinhole orifice and will be projected on the collimator. The divergent radiant beam received within the collimator is then rearranged in parallel and projected in its output as the spectral irradiance of the simulated star. That means the collimator acts as an optical integrator of light. It gets the spectral radiance that propagates in a spherical wave front and transforms it to the spectral irradiance, a plane wave front. Exactly as it is expected from a source of light located at the infinity.

To calculate the spectral irradiance in the collimator output, given the spectral flux produced in the integrating sphere, the equation below was utilized [14]:

$$
L=\left(\frac{\phi_{i}}{\pi A_{s}}\right)\left(\frac{p}{(1-p)\left(1-\left(\frac{A_{i}+A_{e}}{A_{s}}\right)\right)}\right)
$$

Equation 4 supplies the spectral radiance $L\left(\mathrm{~W} \mathrm{~m}^{-2} \mathrm{sr}^{-1}\right.$ $\mathrm{nm}^{-1}$ ) in the integrating sphere output for a given flux $\Phi_{i}$, where $A_{s}$ is the shell area of the integrating sphere, $A_{i}$ is the area of the input door of the integrating sphere, $A_{e}$ is the area of the output door of the integrating sphere and $p$ is the internal reflectance factor of the sphere.

With the equation below it is possible to calculate the spectral irradiance in the collimator output given a spectral radiance at its 
focal plane [14]:

$$
E=2 \pi L A_{p} \frac{1}{8(f / \#)^{2} A_{c}},
$$

where $A_{p}$ is the pinhole area, $A_{c}$ is the area of the beam collimator (input door of the integrating sphere) and $f / \#$ is $f$-number of the collimator. Figure 4 displays the spectral irradiance outside the Earth's atmosphere of a zero magnitude star with a temperature of $7500 \mathrm{~K}$ utilized in the simulations performed in this work.

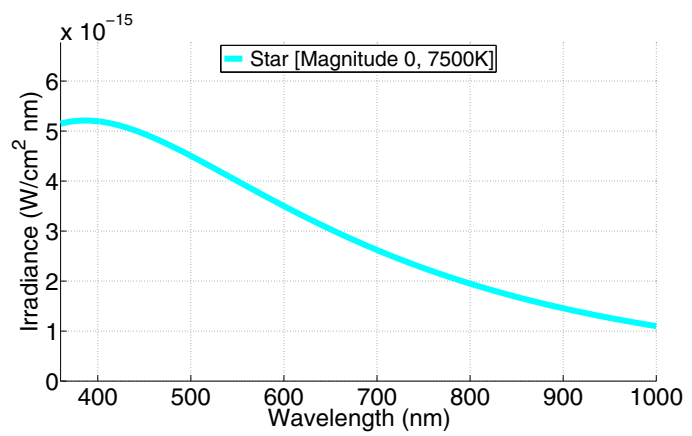

Figure 4 - Spectral irradiance of a zero magnitude star with $7500 \mathrm{~K}$.

\section{CONSTRUCTION OF THE STLS}

The light source could be developed using filament lamps [15], nevertheless it would be impossible to simulate stars above $3500 \mathrm{~K}$ temperature. This source suffers from low flux due to the SPD of the lamp, especially in the ultraviolet and blue region [16]. This means that, to simulate stars of higher temperatures, an alternative would be the use of spectral filters specifically designed for this task. This solution presents the following inconveniences: low luminous efficiency and the heat dissipation of the filament lamp that can deform the collimator, consequently modifying the star relative position during the run. Due to these factors, a LEDbased Spectrally Tunable Light Source was develop.

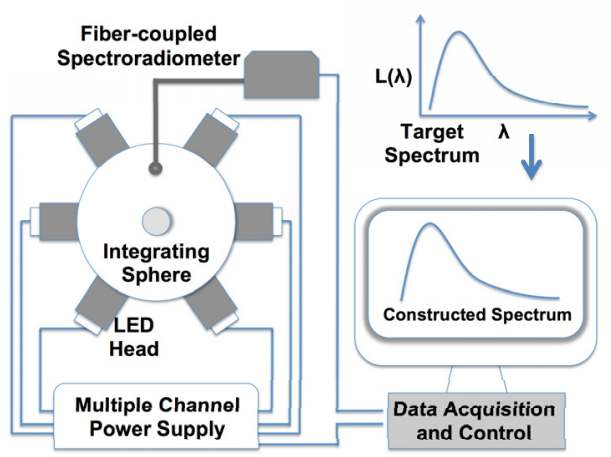

Figure $\mathbf{5}$ - Configuration of the STLS designed and constructed at INPE.

The STLS is a source composed of an integrating sphere illuminated by a large number of LEDs having different spectral peaks and distributions, as represented in Figure 5. The LEDs can be driven and controlled individually with a 96-channel computer controlled power supply. A spectroradiometer is used to monitor the device, in order to determine in real-time, radiometric and photometric output of STLS. The generated data of the spectroradiometer are collected by a computer that individually controls the current of the LEDs, in order to obtain the required target source distribution.

\subsection{Light Emitting Diodes}

By utilizing LEDs with different SPDs and controlling the radiometric output of each type of LED, it is possible to make a spectrally tunable light source capable to produce similar continuum spectra of a specific target with high fidelity [3]. The quality of spectra simulation is intimately linked with the appropriated choice of the LEDs.

The optimal choice of the quantity and type of LEDs for the STLS to be able to simulate stars is known as a multi-objective problem. The main idea here is to minimize the quantity of LEDs and at the same time maximize the spectral matching capability of star spectra.

Taking into account the spectral operation range of the AST, 160 types of LEDs covering the spectral range from 360 to $1000 \mathrm{~nm}$ have been selected and modeled. The difficulty in this process was to obtain the spectral flux of each LED. The datasheets do not bring this information directly, and the information about power is provided on different units, such as candela, watt, watt/spheroradian, and lumens.

Using all technical information available on datasheets, such as radiant diagram, relative luminous intensity diagram, viewing angle, FWHM, radiant power and radiant intensity, the spectral power of each of the candidate LEDs to be used in the STLS was estimated.

\subsubsection{Spectral Power Distribution of a LED}

The SPD of a LED has its base on mathematical models displayed on $[17,18,10,19]$. The SPD of a LED, $S_{L E D}(\lambda)$, for a peak wavelength $\lambda_{0}$ and the full width at half maximum (FWHM) $\Delta \lambda_{0.5}$ is given by

$$
\begin{gathered}
S_{L E D}\left(\lambda, \lambda_{0}, \Delta \lambda_{0.5)}=\right. \\
\frac{g\left(\lambda, \lambda_{0}, \Delta \lambda_{0.5}\right)+2 g^{5}\left(\lambda, \lambda_{0}, \Delta \lambda_{0.5}\right)}{3},
\end{gathered}
$$

where $\left.g\left(\lambda, \lambda_{0}, \Delta \lambda_{0.5}\right)=\exp \left(-\left[\left(\lambda-\lambda_{0}\right) / \Delta \lambda_{0.5}\right)\right]^{2}\right)$. The wavelength is given in nanometers. Figure 6 presents SPD of two 
modeled LEDs compared with the SPD of two typical real LEDs (measured at INPE utilizing a spectroradiometer).
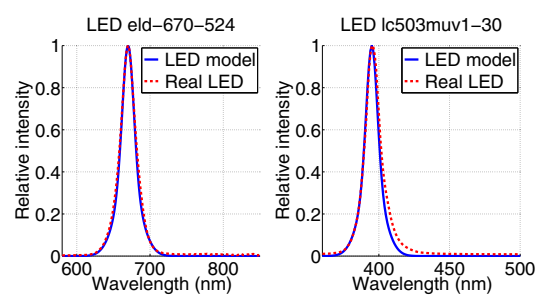

Figure 6 - LED model $S_{L E D}(\lambda)$ compared with the SPD of a typical real LED.

\subsubsection{The angular distribution of a LED}

The intensity of radiation of a LED changes in accordance with the angle of observation, and in general, this information is not afforded by the manufacturer. For the cases in which this information is necessary and is not available in the datasheet of the LED, or in the lack of proper equipment to accomplish the measurement, the approximated intensity distribution of a LED can be modeled by a Super Gaussian [20] given by:

$$
I\left(\theta_{x}, \theta_{y}\right)=I_{0} e^{-2\left(\left(\theta_{x} / \alpha_{x}\right)^{2 G_{x}}+\left(\theta_{y} / \alpha_{y}\right)^{2 G_{y}}\right)},
$$

where $\alpha_{x}$ is the divergent angle between $\mathrm{XZ}$ in degrees, $G_{x}$ is the factor of the Super Gaussian for the direction $X$, with similar definitions for the values of $Y$. Notice that if $G_{x}$ is equal to 1.0 , it results in a typical Gaussian distribution. Either $G_{x}$ or $G_{y}$ must be $\geq 0.01$ and $\leq 50.0$ [20].

For radiation with a Gaussian distribution, the width of spectral band is defined as the length of the radiation range, where the emission power is the half of the maximum power, as can be observed at Figure 7. In the literature this length is known as FWHM. The angle between the central LED position and the position where the emission power of the LED is equal to half of the total power is referenced as $\theta_{f w h m}$ or $2 \theta_{0.5}$ (twice the angle of half power) as exemplified in Figure 8.

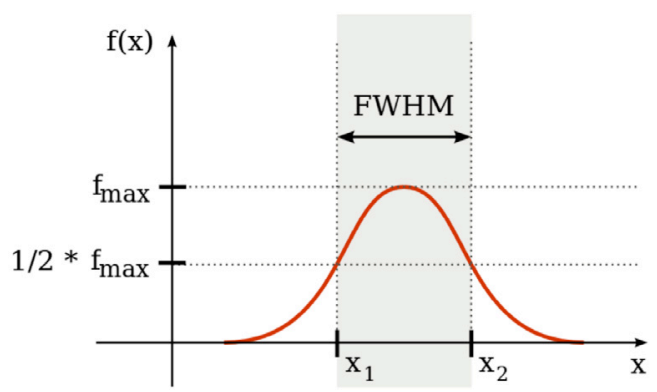

Figure 7 - FWHM for a intensity distribution with Gaussian profile.

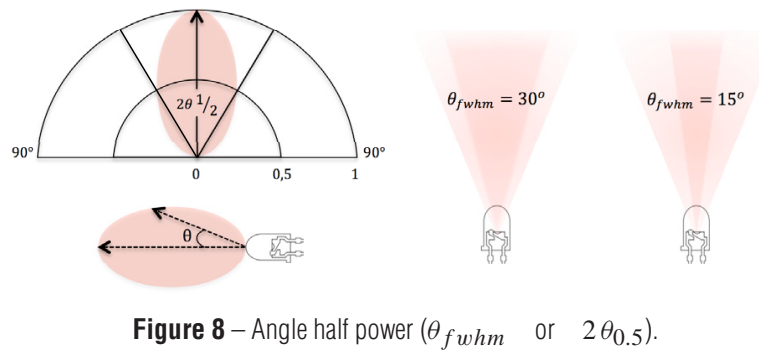

For a typical Gaussian distribution $\left(G_{x}=1\right)$, defining the equation for $\frac{1}{2} I_{0}, \theta_{y}$ equal to zero, substituting the value of $\theta_{x}$ by $\frac{1}{2} \theta_{f w h m}$, then we define $\alpha_{x}$ as:

$$
\alpha_{x}=\frac{\theta_{f w h m}}{\sqrt{2 \ln (2)}} \quad \text { or } \quad \alpha_{x}=(0.8493218) \theta_{f w h m} .
$$

\subsubsection{Luminous flux calculation}

Being the radiation pattern of a LED known in terms of the luminous intensity, its luminous flux $\Phi_{v}$ can be calculated by the following equation:

$$
\Phi_{v}=\int_{0}^{2 \pi} \int_{0}^{\pi} I_{v}(\theta, \phi) \operatorname{sen} \theta d \theta d \phi \quad(\mathrm{Im}),
$$

where $I_{v}(\theta, \phi)$ is the luminous intensity in candela, as a function of a polar angle $\theta$ and an azimuthal angle $\phi$. The angles are always in radians.

When the radiation pattern of a LED is symmetric around the main beam (symmetric in the azimuthal angle $\phi$ ), an unique measure set along of the polar angle $\theta$ is sufficient to describe this pattern. For patterns of symmetrical radiation in $\phi$, Equation 9 can be simplified to the following form:

$$
\Phi_{v}=2 \pi \int_{0}^{\pi} I_{v}(\theta) \operatorname{sen} \theta d \theta \quad(\mathrm{Im}) .
$$

Besides Equations 9 and 10, the luminous flux can also be calculated using the following equation [19]:

$$
\Phi_{v}=K_{m} \int_{\lambda} \Phi_{e}(\lambda) V(\lambda) d \lambda \quad(\mathrm{Im}),
$$

where $K_{m}$ is a constant with a value equal to $683 \mathrm{Im} / \mathrm{W}$ at $555 \mathrm{~nm}$. $\Phi_{e}(\lambda)$ is the spectral distribution of the radiant flux per wavelength $(\mathrm{W} / \mathrm{nm})$, and $V(\lambda)$ is the spectral luminous efficiency function for photopic and scotopic vision [19].

\section{THE MULTI-OBJECTIVE APPROACH}

Optimization problems in engineering are normally of multiobjective nature, frequently presenting objective or conflicting criteria [21, 22, 23]. The objectives should be treated as equally independent, unless there is a function that defines the importance of the relationship between them. The simplest manner for treating 
several objectives is with a transform that adapts a function into a weight average of the several objectives $f=\left[f_{1}, \cdots, f_{k}\right]$ :

$$
g(f)=\sum_{i=1}^{k} W_{i} f_{i} .
$$

The knowledge of the weighs $W_{i}$ introduces, however, a new question: how to quantify the importance of each objective respect to the others. The treatment of some objectives as restrictions is also done. However, one of the perils of this technique is to limit in excess, or in the insufficient manner, the range of solutions.

When a problem is treated as multi-objective, a set of solutions is always found, instead of just a single one. A single solution of this set cannot be considered, in principle, better than any solution in the same set, because at least it will be worse than another solution in one aspect or objective [22, 23]. This set of solutions is known as non-dominated solutions. When these solutions are plotted into the objective function space, they create the, so called, Pareto front. Figure 9 displays the domination and non domination idea of the Pareto front (dots ridge), where the triangles and dots represent solutions of one problem plotted in the space of objective functions, F2 versus F1.

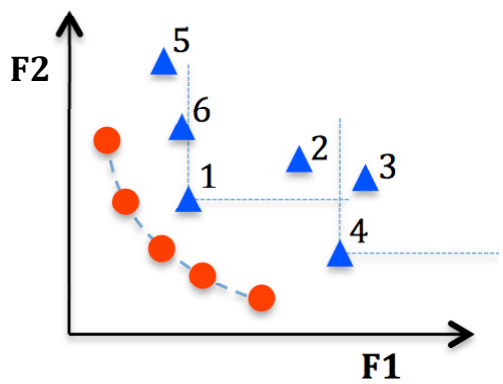

Figure $\mathbf{9}$ - The graph shows solutions for a generic min-min multi-objective problem, plotted in the objective-functions space F1 and F2. Dominated solutions are represented by triangles, while dots represent non-dominated solutions.

To evaluate the characteristics of a solution, a set of metrics called objective functions are utilized. Figure 9 represents a problem of minimization with two objective functions. The lower the values for $\mathrm{F} 1$ and F2, the better is the solution. The dots represent non-dominated solutions and the triangles the dominated ones. One solution $S_{1}$, is considered dominated when there would be another solution $S_{2}$ with at least one of the best objectives better than the objectives of solution $S_{1}$. The dominance connection between dominated solutions can also be considered; for example, the solution 1 dominates the solutions 2 and 3 [22, 23].

The aim of a multi-objective problem is to find non-dominated solutions that generates the Pareto front. Thus, after the process of multi-objective optimization, a set of obligation solutions are obtained, and not only a single solution as happens in a monoobjective approach. This allows designers a clear idea of the obligations that can be made in the project considering the metrics utilized.

The evolutionary optimization algorithm is a tool to resolve directly multi-objective optimization. In this work we made use of a multi-objective version of the Generalized Extremal Optimization algorithm (M-GEO).

\subsection{Generalized Extremal Optimization and its variations}

Concerning optimization problems, there is a large probability that the algorithm applied in the search for optimal solutions stays attached to a local minimum. To avoid that, several methods were developed that accomplish a global search for the optimal solution in project space $[22,23]$.

One class of these methods was constructed inspired by processes that occur in nature, that is, deals with the problems of optimization of numerical mechanisms that try to mimic natural phenomena. The Genetic Algorithm (GA) and the Simulated Annealing $(S A)$ are examples of those methods that stand out in this class of algorithms. The GA and SA have been developed and applied to a large number of optimization problems, both in science and in engineering.

Recently, a new global search method inspired on nature was proposed by Sousa [24], the Generalized Extremal Optimization algorithm (GE0). This method was improved by Galski [25], that proposed variations of GEO such as GEO real and M-GEO (a multi-objective version of GEO).

\subsubsection{Canonical GEO}

The GEO was presented by Sousa et al. [24] as a generalization of the Extremal Optimization algorithm (EO) proposed by Boettcher [26]. Both are based in the Self-Organized Criticality theory by Bak and Sneppen [27].

The codification of the design variable is binary, as well as the encoding used in the GA. However, the string of bits does not represent the chromosome of one candidate solution, but different species coexisting in one ecosystem as it can be observed in Figure 10. The GEO owns some advantages above other evolutionary algorithms: it has only one free parameter for adjustment, its implementation is very simple and it has shown a competitive performance for test functions in real situations $[24,28,29,25,30,31,32]$. Figure 11 displays the diagram of the GEO. 


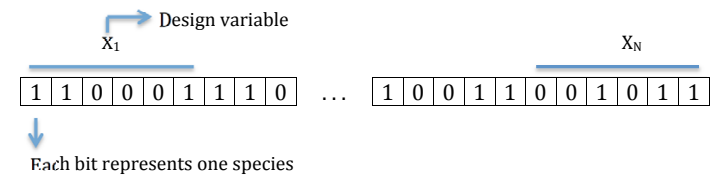

Figure 10 - Design variables encoded in a binary string; in this example each variable is represented by six bits.

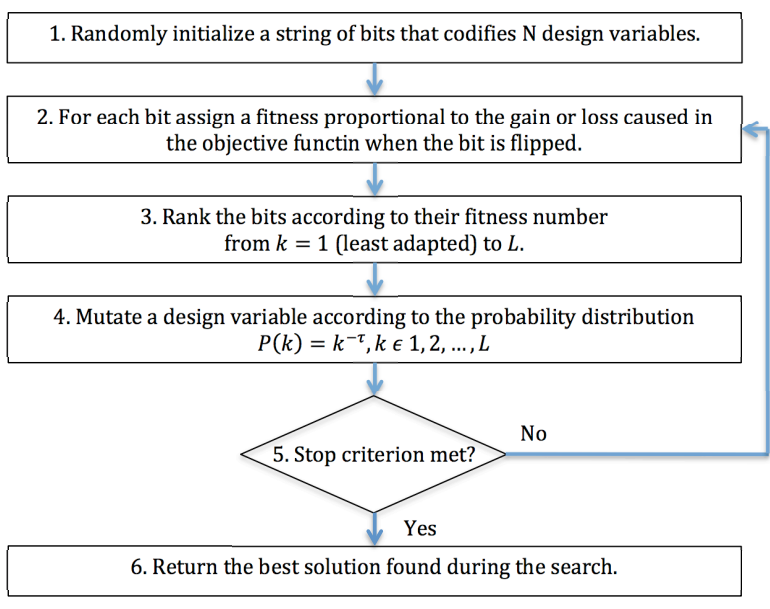

Figure 11 - Canonical GEO flowchart candidate solution representation.

For each bit of the sequence an index of adaptability is attributed which shows the gain or the loss that the value of the objective function has when it suffers mutation (changes the value 0 to 1 or 1 to 0 ). The next step is to sort the bits of $k=1$ to $k=L$, where the bit with minor adaptability receives the index 1 and the most adapted receives L. Following one bit is selected and it suffers mutation with the uniform probability described by the following equation:

$$
P(k)=k^{-\tau}, \quad 1 \leq k \leq N,
$$

where $\tau$ is a positive adjustable parameter. This method is repeated until a given stopping criterion is satisfied. The best bit configuration (solution) found during the search is saved and is given in the end of the run.

\subsubsection{Multi-Objective GEO - M-GEO}

In the past years several variations of the canonical GEO were suggested. These variations also present competitive results, both for tests, and real problems [25, 33].

Galski presented the multi-objective version of GEO, called M-GEO [25]. The principal implemented evolution of the multiobjective algorithm version occurs in the bits fitness calculation. For each generation in the M-GEO, one objective function among the $\mathrm{N}$ objective functions of the problem is selected with uniform distribution and utilized to conduct the search. Another change is that at each generation the solutions are analyzed, and all nondominated solutions are saved, generating the Pareto front as the output. Figure 12 shows the M-GEO diagram.

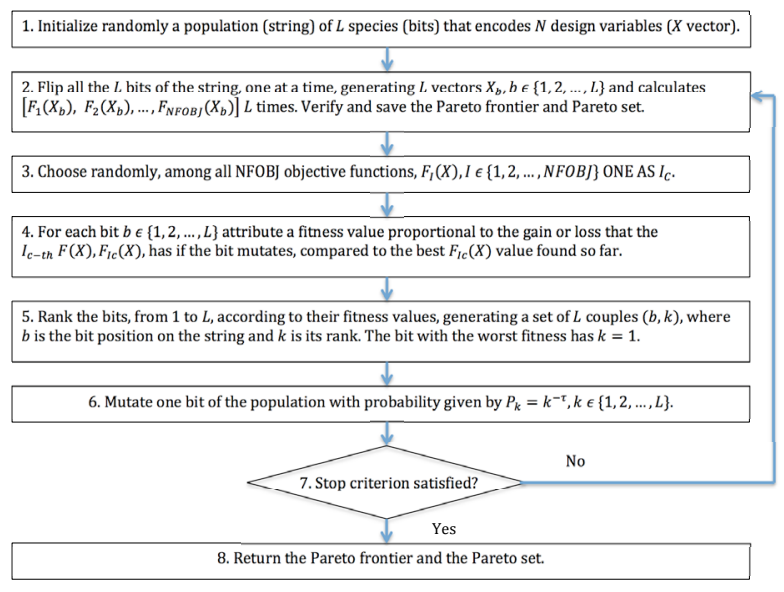

Figure 12 - M-GEO flowchart.

\section{METHODOLOGY}

This section provides step-by-step run for the modeling of LEDs, setting of the stars to be simulated, and configuring the M-GEO.

\subsection{Modeling of LEDs}

Initially, several LEDs covering the spectral range 360-1000 nm were selected. The selected LED datasheet was acquired from the manufacturer, for extracting important data in the modeling of LEDs. There is no standard in the terms used in the datasheets, hampering the process for identifying and collecting information.

In this work, were compiled from each LED, when available on datasheet, values for luminous intensity (mcd), view angle (degrees), peak wavelength $(\mathrm{nm})$, FWHM $(\mathrm{nm})$, radiant power $(\mathrm{mW})$ and radiant intensity (mW/sr).

The modeling was initially performed by calculating the normalized SPD for each LED, $S_{L E D}(\lambda)$, using the equation 6 . Then, the intensity distribution of radiation angle, $I\left(\theta_{x}, \theta_{y}\right)$, was calculated using the equations 7 e 8 . To calculate the actual SPD of each LED it was necessary to calculate the conversion factor, $F_{L E D}$, which represents the multiplying factor to convert the normalized SPD to real SPD.

Some LEDs bring in their datasheet the radiant power information $\Phi_{e}$ in $\mathrm{mW}$. For these LEDs, the conversion factor $F_{L E D}$ was calculated based on Equation 11 as follows:

$$
F_{L E D}=\frac{\Phi_{e}}{\int_{\lambda} S_{L E D}(\lambda) d \lambda} .
$$

For LEDs that show only the luminous intensity in their 
datasheets, the luminous flux was calculated using the equations 9 and 10, and the conversion factor $F_{L E D}$ calculated as follows:

$$
F_{L E D}=\frac{\Phi_{v}}{K_{m} \int_{\lambda} S_{L E D}(\lambda) V(\lambda) d \lambda} .
$$

For LEDs that show just radiant intensity in their datasheets, the radiant flux was calculated using the equations 9 and 10 and conversion factor $F_{L E D}$ was calculated using Equation 13. After this process, having calculated the conversion factor $F_{L E D}$, the real SPD for each selected LEDs was calculated as follows:

$$
S P D_{L E D}(\lambda)=S_{L E D}(\lambda) F_{L E D} .
$$

Figure 13 shows the SPDs of 160 candidates LEDs to be used in the STLS.

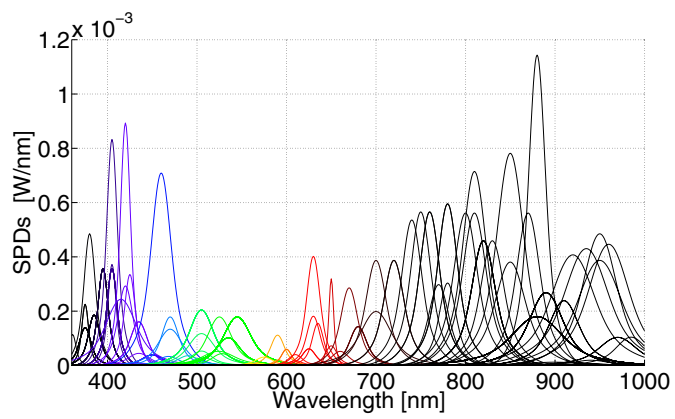

Figure 13 - SPD of 160 candidates LEDs.

\subsection{Optimizing the choice of LEDs}

The STLS was designed to simulate the brightest stars, of magnitude zero, until the less bright stars, of magnitude five. Using the equations 1, 2 and 3a, the spectrum of all stars that STLS should simulate, was constructed. To appropriate choice of LEDs, the sufficient power to simulate all stars of magnitude zero must be guaranteed. Getting power to simulate the brightest stars, of magnitude zero, the power to simulate the less bright stars is also ensured. Figure 14 displays the spectral irradiance that encompasses all stars of magnitude zero, selected from the Hipparcos Stars Catalog.

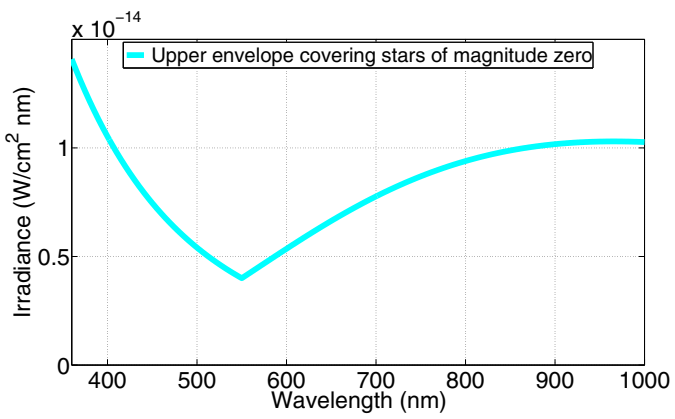

Figure 14 - Spectral irradiance covering stars of magnitude zero.
The spectrum presented in Figure 14 was used as a reference for the appropriate choice os LEDs. The choice of LEDs for the spectral irradiance covering all stars makes it possible for the STLS to simulate the brightest stars, of magnitude zero, until the less bright, of magnitude five.

After modeling the LEDs and defining the spectrum of the target star to be simulated, a simulator for STLS was implemented. The simulator was designed to have the ability to mimic the spectrum generated by LEDs with different power in the collimator output.

The choice of the amount and types of LEDs was initially tried with Levenberg-Marquardt algorithm (LM). The solution achieved with LM suggested a huge amount of LEDs, which precluded the construction of STLS, since there is no space for more than 200 LEDs in the integrating sphere. Figure 15 shows the fiting achieved with LM to spectral irradiance presented in Figure 14. Unfortunately the amount of 1202 LEDs was unfeasible to build the STLS.

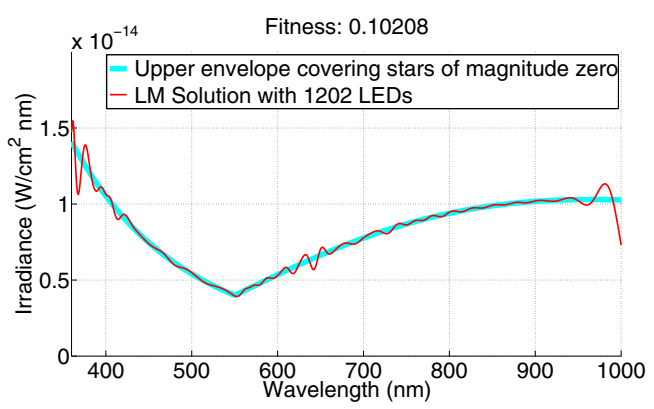

Figure 15 - Fiting with Levenberg-Marquardt.

The LM is an optimization method that searches the local minimum in a function, and generally converges faster than a GA, however biases the sub-optimal solutions and in some cases do not converge. To make it possible the build the STLS it is necessary to minimize the fitting error and also the number of LEDs. For this multi-objective optimization task, the multi-objective version of GEO, the M-GEO, was chosen.

The M-GEO algorithm was coded in $\mathrm{C}_{++}$using the open source framework Qt. To improve the performance of M-GEO and use all available computational resource, the algorithm has been implemented to perform the calculation of the objective function in parallel. The source code of the M-GEO will be available in https://github.com/marcosegborges.

In M-GEO, each modeled LED was treated as a design variable. The minimum value for each variable $i$, was set to $X_{\min } i=$ 0 , and maximum value to $X_{\max } i=100$. The value for the precision of each variable was defined as $e_{i}=0.1$. The parameter setting for M-GEO, $\tau=3$, was obtained empirically. 
Another important aspect is the definition of the number of bits of each variable, $l_{i}$, based on the minimum precision desired for each variable. The following equation was used to calculate the smallest number of bits needed to represent each variable $[24,25]$ :

$$
l_{i}=\log _{2}\left(1+\frac{\left(X_{\max } i-X_{\min } i\right)}{e_{i}}\right) .
$$

The M-GEO initialization fills a binary string $C$ of length $L$, through $L$ sweepstakes of 0 s and 1 s, each with equal probability of occurrence. This defines a binary string configuration, or candidate solution to global optimal, represented in numerical system used internally by M-GEO (binary system). The numerical system used to represent the design variables is not binary, but decimal. In order to calculate the objective function, $F(X)$, it is necessary to first convert $\mathrm{C}$ in $\mathrm{X}$, in other words, convert each design variable, $X_{i}, i \in\{1,2, \ldots, N\}$, its binary encoding to its corresponding decimal value using the following equation [24, 25]:

$$
X_{i}=X_{\min } i+\left(X_{\max } i-X_{\min } i\right) \frac{\sum_{j=1}^{l_{i}} C_{j} 2^{j-1}}{2^{l_{i}}-1} .
$$

Using Equation 17 we obtain the decimal value for each variable of candidate solution to global optimum. The decimal value of each variable represents the power percentage of each LED, where a value of 0.3 represents $30 \%$ of the LED, and a value of 2.4 represents two LEDs in $100 \%$ and the third in $40 \%$.

To solve the multi-objective problem presented in this work, two objectives were defined: minimization of the total number of LEDs and the minimization of the fitting error. The following equation was used to calculate the fitting error:

$$
\begin{aligned}
& \text { Error }_{\text {fiting }}= \\
& \sqrt{\sum_{\lambda=360}^{1000}\left(\sum_{i=1}^{160} E \text { Power }_{L E D_{i}} \operatorname{SPD}_{L E D_{i}}(\lambda)-M(\lambda)\right)^{2}},
\end{aligned}
$$

where $E$ is the system transfer function of the integrating sphere and collimator calculated according to Equations 4 and 5, Power $_{L E D_{i}}$ is the power of the LED type $i$ (real value), $S P D_{L E D_{i}}(\lambda)$ is the radiant flux of the LED per wavelength and $M(\lambda)$ is the spectral irradiance per wavelength desired in the collimator output.

The total number of LEDs was calculated using the following equation:

$$
\operatorname{Total}_{L E D s}=\sum_{i=1}^{160}\left\lceil\operatorname{Power}_{L E D_{i}}\right\rceil .
$$

\section{RESULTS AND DISCUSSION}

After the acquisiton of technical information and modeling of LEDs, integrating sphere, and pinhole, definition of the collimator transfer function, modeling of the star to be simulated, and the definition of the objective functions, the M-GEO algorithm implemented in $\mathrm{C}_{+}+$was run on Windows 64 bit, Intel i7-3770 3.40 GHz with $8 \mathrm{~GB}$ of RAM. Figure 16 shows the Pareto front obtained by running the algorithm for the reference star spectrum, consuming approximately 77 hours.

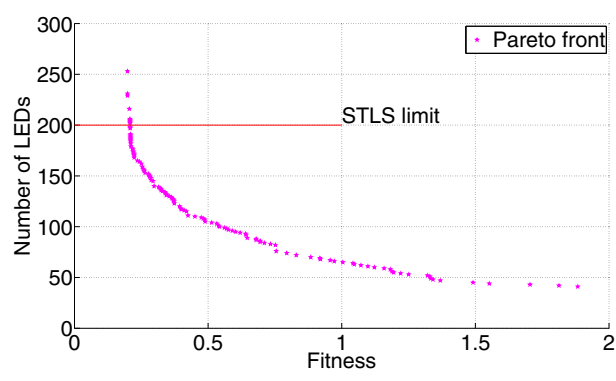

Figure 16 - Pareto Front achieved by M-GEO.

As expected, the algorithm produced different feasible solutions, allowing designers to choose the best solution according to the limitations of the project. Analyzing the Pareto front and considering the solutions below the limit of the number of LEDs of the STLS, there are several viable solutions of 175-200 LEDs with very similar fitness. Figures $17(\mathrm{a})$ and $17(\mathrm{~b})$ show solutions with the amount of LEDs over the limit of the STLS.

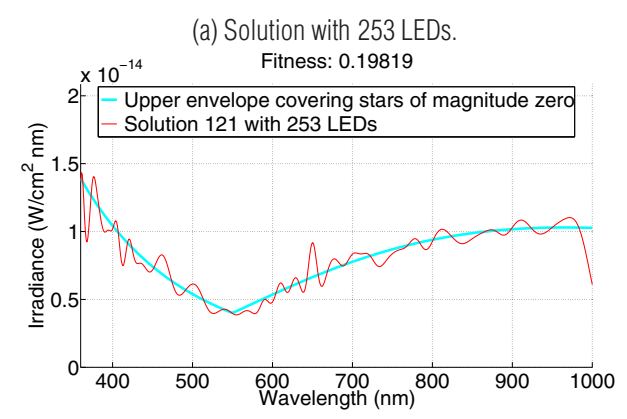

(b) Solution with 229 LEDs.

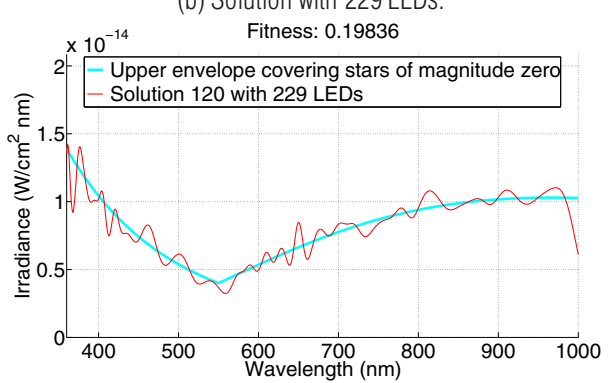

Figure 17 - Two solutions generated by M-GEO. The number of LEDs is over the STLS limits. 
The fitness value was calculated using Equation 18. The lowest is the value of the fitness, the best is the solution. Figures 18(a) and 18 (b) show solutions with viable number of LEDs. The fittings generated by solutions with 253 and 229 LEDs are very similar to those generated by the solutions with 198 and 174 LEDs.

(a) Solution with 198 LEDS.

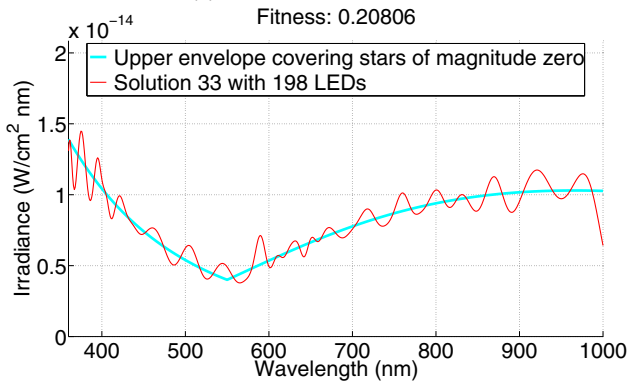

(b) Solution with 174 LEDs.

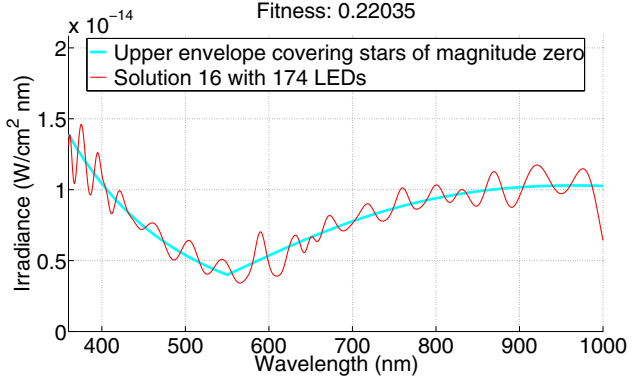

Figure 18 - Two solutions generated by M-GEO. The number of LEDs is over the STLS limits.

The choice for the construction of the STLS was with 198 LEDs. The maximum number of LEDs that fits in the integrating sphere is 200. Other solutions could have been chosen. However, all solutions below the solution with 198 LEDs have worse fiting. Before the purchase of the LEDs, several simulations were performed to validate the solution achieved by the M-GEO. Figure 19 shows the simulation results for a star of magnitude zero with $7500 \mathrm{~K}$. Although the fiting is not perfect, the small difference between the desired and the simulated spectrum will not interfere in the simulations of stars for calibration of AST.

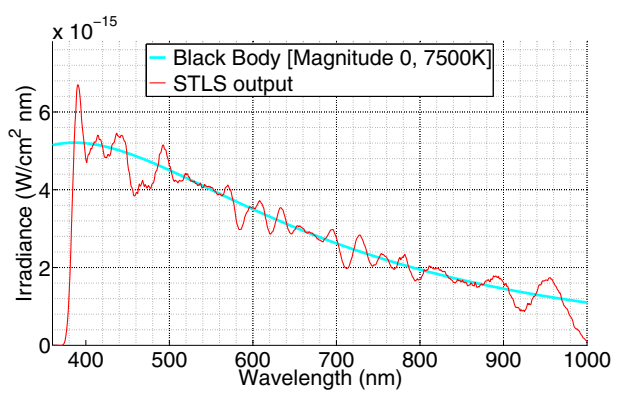

Figure 19 - Simulation of STLS for a star of magnitude zero with $7500 \mathrm{~K}$.
Figure 20 shows the simulation results for a star of magnitude zero with $20000 \mathrm{~K}$. The simulation of this star had a behavior similar to Figure 19, with a poor fiting in 480-490 nm.

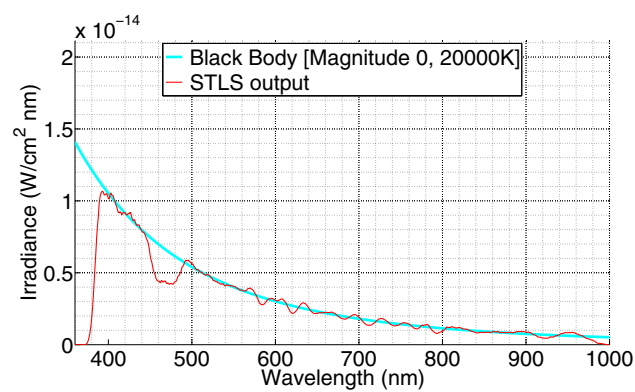

Figure 20 - Simulation of STLS for a star of magnitude zero with $20000 \mathrm{~K}$.

Figure 21 shows the simulation results for a star of magnitude zero with $5600 \mathrm{~K}$. The fitings are not perfect due to the limited amount of LEDs imposed upon STLS. For better results it would be necessary to build a light source with a larger LEDs capability.

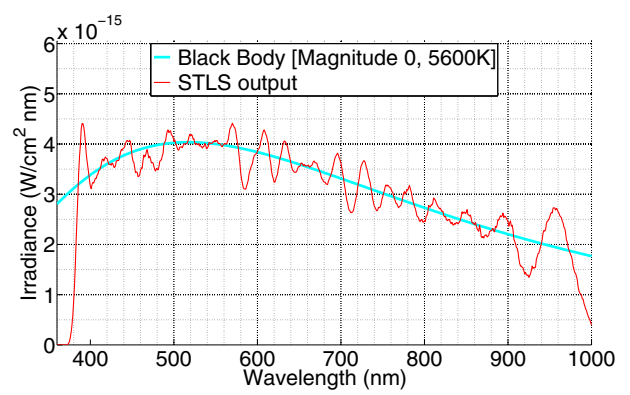

Figure 21 - Simulation of STLS for a star of magnitude zero with $5600 \mathrm{~K}$.

\section{CONCLUSION}

In this work, we presented the methodology applied in multiobjective optimization for choosing the amount and types of LEDS required for the Star Simulator. Initially, classical methods were tentatively used to solve the problem. However, viable results have not been achieved, given the dimensions and engineering constraints of the STLS. Thus, we sought a solution with an evolutionary algorithm M-GEO, which showed satisfactory results.

M-GEO is inspired in Self-Organized Criticality theory, and fits in the category of optimization methods inspired by nature, such as SA and GA. One of the advantages that the M-GEO has on the $S A$ and GA is that it has only one tuning parameter and its implementation is very simple. The performance of an evolutionary algorithm on a given problem is dependent on the proper fit of its free parameters, and in some cases adjusting these parameters can become a problem on itself.

Several simulations were performed to evaluate the performance of STLS for the selection of the number and type of LEDs. 
The simulation results indicate that the STLS reasonably satisfies the simulation requirements of stars necessary for calibrating the AST.

\section{ACKNOWLEDGMENTS}

The authors acknowledge the financial support provide by CNPq and INPE to make possible the realization of this work.

\section{REFERENCES}

[1] WERTZ JR. 1978. Spacecraft Attitude Determination and Control, Microcosm Inc, Torrance, CA, USA.

[2] WERTZ JR \& LARSON WJ. 1999. Space Mission Analysis and Design, 3rd edn, Microcosm Press, Hawthorne, CA 90250, USA.

[3] FRYC I, BROWN SW \& OHNO Y. 2005. Spectral matching with an LED-based spectrally tunable light source, Vol. 5941, SPIE, p. 594111.

[4] de ALBUQUERQUE BFC. 2005. Estudo dos erros sistemáticos inerentes a um sensor de estrelas de cabeça fixa, quando à Iocalização relativa de estrelas, Master's thesis, Instituto Nacional de Pesquisas Espaciais (INPE), São José dos Campos.

[5] FIALHO MAA. 2007. Estudo comparativo entre dois algoritmos de identificação de estrelas para um sensor de estrelas autônomo de campo largo, Master's thesis, Instituto Tecnológico de Aeronáutica (ITA), São José dos Campos.

[6] ALLEN CW. 1973. Astrophysical quantities, William Clowes, London.

[7] HALLIDAY D, RESNICK R \& KRANE KS. 1996. Física 4, LTC Livros Técnicos e Científicos Editora S.A., Rio de Janeiro.

[8] SMITH WJ. 1996. Modern Optical Engineering: The Design of Optical Systems, McGraw-Hill, USA.

[9] SMITH WJ. 2007. Modern Optical Engineering, McGraw-Hill, USA.

[10] SCHUBERT EF. 2006. Light-Emitting Diodes, 2nd edn, Cambridge University Press, UK.

[11] ESA. 1997. The hipparcos and tycho catalogues. (ESA SP-1200).

[12] CDS. 2007. The hipparcos and tycho catalogues. (CDS I/239).

[13] REED BC. 1998. The composite observational-theoretical HR diagram. Journal of the Royal Astronomical Society of Canada, 92: 36-37.

[14] LABSPHERE. 2012. A Guide to Integrating Sphere Radiometry and Photometry, US.

[15] WALL CF, HANSON AR \& TAYLOR JAF. 2001. Construction of a programmable light source for use as a display calibration artifact. Proc. SPIE, 4295: 259-266.
[16] FRYC I, BROWN SW, EPPELDAUER GP \& OHNO Y. 2005. Ledbased spectrally tunable source for radiometric, photometric, and colorimetric applications. Optical Engineering, 44: 111309.

[17] OHNO Y. 2004. Color rendering and luminous efficacy of white led spectra, Vol. 5530. SPIE, pp. 88-98.

[18] OHNO Y. 2005. Spectral design considerations for white led color rendering. Optical Engineering, 44: 111302.

[19] CIE. 2007. CIE 127:2007: measurement of LEDs (2nd ed), Technical report, Vienna, Austria.

[20] ZEMAX. 2011. Zemax Optical Design Program: User's Manual, Tucson.

[21] VANDERPLAATS GN. 1998. Numerical optimization techniques for engineering design, 2nd edn, Vanderplaats Research \& Development, Colorado Springs.

[22] EIBEN AE \& SMITH JE. 2007. Introduction to Evolutionary Computing, Springer, Berlin.

[23] BRANKE J, DEB K, MIETTINEN K \& SLOWINSKI R. 2008. Multiobjective Optimization: Interactive and Evolutionary Approaches, Springer-Verlag, Berlin.

[24] de SOUSA FL, RAMOS FM, PAGLIONE P \& GIRARDI RM. 2003. New stochastic algorithm for design optimization. AIAA Journal, 41: 1808-1818.

[25] GALSKI RL. 2006. Desenvolvimento de versões aprimoradas híbridas, paralela e multiobjetivo do método da otimização extrema generalizada e sua aplicação no projeto de sistemas espaciais, PhD thesis, Instituto Nacional de Pesquisas Espaciais (INPE), São José dos Campos.

[26] BOETTCHER S \& PERCUS A. 2001. Optimization with extremal dynamics. Physical Review Letters, 86: 5211-5214.

[27] BAK P \& SNEPPEN K. 1993. Punctuated equilibrium and criticality in a simple model of evolution. Physical Review Letters, 71 : 4083-4086.

[28] de SOUSA FL, VLASSOV V \& RAMOS FM. 2004. Generalized extremal optimization: An application in heat pipe design. Applied Mathematical Modelling, 28: 911-931.

[29] ABREU B, MARTINS E \& de SOUSA FL. 2005. Automatic test data generation for path testing using a new stochastic algorithm. In: Proceedings of 19th Brazilian Symposium on Software Engineering (SBES) pp. 247-262.

[30] SWITALSKI P \& SEREDYNSKI F. 2008. Generalized extremal optimization for solving multiprocessor task scheduling problem. Simulated Evol. and Learning Lecture Notes in Comp. Science, 5361: 161-169.

[31] SWITALSKI P \& SEREDYNSKI F. 2011. A grid scheduling based on generalized extremal optimization for parallel job model. Parallel Processing and Applied Mathematics, 7204: 41-50. 
[32] SWITALSKIP \& SEREDYNSKIF. 2012. An effective multiprocessor scheduling with use of geo metaheuristic. International Journal of Foundations of Computer Science, 23: 465-481.
[33] LOPES IML, de SOUSA FL \& de SOUZA LCG. 2008. The generalized extremal optimization with real codification, International Conference on Engineering Optimization. 